\title{
Serial-pattern-learning processes dissociated by trimethyltin exposure in rats
}

\author{
STEPHEN B. FOUNTAIN, DAVID E. SCHENK, and ZOLTAN ANNAU \\ Department of Environmental Health Sciences, The Johns Hopkins University, Baltimore, Maryland
}

\begin{abstract}
Trimethyltin (TMT) is a neurotoxic organometal which produces a variety of learning and memory impairments in laboratory animals and humans, including impairments of avoidance learning, maze learning, and problem solving. Two studies investigated the effects of TMT exposure on serial-pattern learning in rats. Rats in both experiments were intubated once with either 0 or $7.0 \mathrm{mg} / \mathrm{kg}$ TMT 1 week prior to the pattern-learning procedure. Rats learned serial patterns composed of various quantities of brain-stimulation reward (BSR) pulses; they received BSR quantities in a predetermined order for leverpresses in a discrete-trial operant task. In Experiment 1, all rats received two serial patterns (20-10-0 vs. 1-29-0 pulses of BSR) that alternated within each daily session of 100 patterns. In Experiment 2, all rats received two serial patterns (18-10-6-3-1-0 vs. 18-1-3-6-10-0 pulses of BSR) that alternated within each daily session. In Experiment 1, TMT-exposed rats learned both their patterns more slowly than did controls. In Experiment 2, TMT-exposed rats learned the formally simple 18-10-6-3-1-0 pattern of BSR quantities faster than did controls, but were significantly slower than controls in learning the formally more complex 18-1-3-6-10-0 pattern. In both experiments, however, TMT exposure did not affect either the reinforcing properties of BSR or rats' asymptotic performance. These results support the notion that TMT exposure impaired some aspects of the rote processes involved in serialpattern learning in rats, yet spared the rats' ability to encode some representation of the formal rule-based structure of the pattern. The results thus suggest that the processes involved in learning simple versus complex serial patterns may be mediated by different systems in the brain.
\end{abstract}

Trimethyltin (TMT) is a neurotoxic compound that has been shown to produce impairments of learning and memory in both animals and humans. Neuronal damage resulting from TMT exposure in rats is most extensive in the hippocampus, though limited neuropathology has been found in the pyriform cortex, amygdala, and neocortex (Brown, Adridge, Street, \& Verschoyle, 1979; Valdes, Mactutus, Santos-Anderson, Dawson, \& Annau, 1983). Behavioral effects in rats associated with TMT toxicity include hyperactivity (Ruppert, Walsh, Reiter, \& Dyer, 1982), hyperreactivity (Dyer, Walsh, Wonderlin, \& Bercegeay, 1982), impaired retention in passive avoidance tasks (Walsh, Gallagher, Bostock, \& Dyer, 1982), and deficits in maze performance (Swartzwelder et al., 1982; Walsh, Miller, \& Dyer, 1982). We investigated the effects of TMT exposure on rat serial-pattern learning, a task that can be designed so as to encourage rats to utilize either classical associative strategies for pattern learning that place great demands on rote processes or, in contrast, more cognitive strategies such as rule en-

This research was supported by National Institutes of Health Research Service Award MH 08759 from the National Institute of Mental Health to S. B. Fountain, and by ES 07094 and ES 02277 to Zoltan Annau. We thank Robert E. Lintz and Beth L. Wilhite for technical assistance. We also thank Maria S. Zaragoza for her comments on an earlier version of the article. Reprints may be obtained from Stephen B. Fountain, Department of Neurobiology, Northeastern Ohio Universities College of Medicine, Rootstown, $\mathrm{OH} 44272$. coding that place greater emphasis on abstraction and information organization. Because TMT is considered a relatively selective limbic system neurotoxicant, we expected that rats' performance on pattern-learning tests following TMT exposure would shed light on limbic system involvement in the rote and rule-based processes mediating rat sequential learning.

Because all behavior is sequentially organized in time, serial learning is a fundamental problem for both humans and animals. When events appear in a regular pattern in time, appropriate anticipation of future events in the pattern requires that the organism learn ordered relationships among the events that make up the pattern. Functional similarities between human and rat serial-pattern-learning processes have already been demonstrated. For example, under appropriate conditions, rats can use complex cognitive strategies for learning serial patterns that include learning rules to encode the structure of patterns (Hulse \& Dorsky, 1977) and "chunking" patterns into meaningful units to facilitate learning (Fountain \& Annau, 1984; Fountain, Henne, \& Hulse, 1984). Humans and rats appear to use similar cognitive processes in learning serial patterns.

Two experiments tested the hypothesis that TMT exposure would adversely affect serial-pattern learning in rats. In both experiments, rats first learned to leverpress for brain-stimulation reward (BSR). After exposure to either 0 or $7.0 \mathrm{mg} / \mathrm{kg}$ TMT, rats were tested again in a simple leverpressing task to test the effect of TMT on rats' 
response to the rewarding quality of brain stimulation and the effect of TMT on resistance to extinction. Rats then received two patterns made up of quantities of BSR arranged in a consistent order; the patterns alternated throughout testing. On the basis of pilot work, we expected that TMT exposure would not affect the reinforcing properties of BSR. We assumed that TMT-induced deficits in the processes mediating serial-pattern learning would be reflected in disrupted acquisition when rats learned their serial patterns.

\section{EXPERIMENT 1}

In Experiment 1, each rat received two patterns made up of various quantities of BSR. A monotonically decreasing 20-10-0 pattern of BSR quantities alternated with a nonmonotonic 1-29-0 pattern. Each quantity that made up the patterns was delivered on a separate trial as reward for a single leverpress. Response latency was measured on each trial and was assumed to indicate rats' anticipation of reward magnitude. Previous research had shown that rats will learn to track (Hulse, 1978) sequential patterns, responding fast in anticipation of large quantities of BSR and slowly, or not at all, in anticipation of small quantities of BSR in this type of procedure (Fountain \& Annau, 1984). Differentiation of response latency in anticipation of large and small quantities was used as a measure of rats' rate of pattern learning. Most important in this regard was rats' response to nonreward (the 0-pulse BSR quantity), because rats initially respond fast for all quantities of their patterns and only later learn to slow their response in anticipation of the smallest quantities of BSR (Fountain \& Annau, 1984).

\section{Method}

Subjects. The subjects were 10 naive male hooded rats obtained from Blue Spruce Farms, Altamont, New York. The rats weighed approximately $300 \mathrm{~g}$ at the time of implantation of electrodes, and weighed approximately $350 \mathrm{~g}$ at the beginning of the experiment. The rats were housed in individual plastic tubs, and were provided free access to food and water in their home cages throughout the experiment. One rat died 3 days following intubation with TMT and one rat was excluded from the experiment because of damage to the electrode assembly that occurred prior to the serial-patternlearning phase of the experiment. Thus, four rats in each of two conditions completed the experiment. The rats were weighed each day except during the 4-day recovery period following intubation. On testing days, the rats were weighed approximately $30 \mathrm{~min}$ prior to testing.

Apparatus. Operant chambers $(30 \times 30 \times 30 \mathrm{~cm})$ were composed of clear Plexiglas walls and a floor of stainless steel rods $(0.5 \mathrm{~cm}$ in diameter) spaced $1.25 \mathrm{~cm}$ apart. One end wall was equipped with a response lever located $4.0 \mathrm{~cm}$ from the front wall and $5.0 \mathrm{~cm}$ above the floor. Rats in the testing chamber were connected to a stimulator by way of a flexible cord (Plastic Products MS304) and a commutating device that allowed the animal free movement within the chamber. The operant chamber was enclosed in a sound-attenuating shell $(55 \times 50 \times 55 \mathrm{~cm})$ made of particle board. A houselight (7.5 W) was centered approximately $5 \mathrm{~cm}$ below the ceiling on the rear wall of the shell. The experiment was controlled from an adjoining room by a Digital Equipment Corpo- ration PDP-8/e minicomputer and SKED software system (State Systems, Inc.; Kalamazoo, Michigan).

Implantation and initial training. Each rat was anesthetized by intraperitoneal Chloropent injection $(3.33 \mathrm{cc} / \mathrm{kg})$. A twisted stainless steel bipolar electrode (Plastic Products MS303/1) was then implanted unilaterally in the posterior lateral hypothalamus (coordinates with the skull level: $4.5 \mathrm{~mm}$ posterior to the bregma, $1.5 \mathrm{~mm}$ lateral to the midline, and $8.5 \mathrm{~mm}$ below the surface of the skull). After 5-7 days of recovery time, each rat was placed in an operant chamber and connected to a stimulator by way of a flexible cord and a commutating device. The rats were shaped to leverpress for BSR composed of a single $100-\mathrm{msec}$ pulse of a $60-\mathrm{Hz}$ sinusoidal pulse train from a constant current source of 20-40 $\mu \mathrm{A}$. Training sessions lasted $30 \mathrm{~min}$ on 2 consecutive days. At this time, the stimulating current was adjusted so that each rat produced at least 3,000 leverpresses in $30 \mathrm{~min}$ of training. The rats that failed to meet this requirement were excluded from the experiment, resulting in 10 rats' continuing to the next phase of training.

Light-dark discrimination pretraining. For 2 days following initial training, the rats were trained to discriminate light-on and light-off periods in the operant chamber. Throughout a 30 -min session, the houselight was alternately illuminated for $2 \mathrm{~min}$ and extinguished for $15 \mathrm{sec}$. When the houselight was illuminated, leverpresses were reinforced. When the houselight was extinguished, leverpresses were never reinforced.

Intubation. Approximately $1 \mathrm{~h}$ following training on the 2 nd day of light-dark discrimination training, half the rats were intubated with $0 \mathrm{mg} / \mathrm{kg}$ (control) and half with $7.0 \mathrm{mg} / \mathrm{kg}$ (TMT-exposed) TMT-chloride in saline solution. The volume of solution administered to each rat was equivalent to $3 \mathrm{cc} / \mathrm{kg}$.

Light-dark discrimination testing. On Days 5 and 6 following intubation, the rats were tested on the same light-dark discrimination already described.

Serial-pattern learning. On the day immediately following the conclusion of light-dark discrimination training, the rats received different quantities of BSR pulses for leverpresses on consecutive trials. The BSR quantities were arranged in two patterns, and all rats received the quantities in the same order each day. The rats received a monotonically decreasing 20-10-0 pattern of BSR quantities as the first pattern each day, followed by a nonmonotonic 129-0 pattern of BSR quantities. Thereafter, the two patterns alternated throughout the daily session. Each trial that made up the patterns began when the houselight was turned on and continued until the rat leverpressed or until $15 \mathrm{sec}$ had elapsed. If the rat leverpressed within $15 \mathrm{sec}$, the appropriate quantity of BSR was delivered. For example, on successive trials of the first pattern each day, leverpresses while the light was on produced first 20 pulses of BSR, then 10 pulses of BSR, and then 0 pulses of BSR. BSR quantities were $100-\mathrm{msec}$ pulses of stimulation separated by $100 \mathrm{msec}$. Trials were terminated after a 15 -sec nonresponse criterion was met. No BSR was delivered on trials in which rats failed to leverpress. The houselight was extinguished for $1 \mathrm{sec}$ between trials and for $1 \mathrm{~min}$ between patterns. A response latency, the time elapsed between the beginning of the trial and the rat's leverpress, was recorded for each trial to the nearest $0.01 \mathrm{sec}$. A 15 -sec latency was recorded for trials in which the rat failed to respond. Rats received 50 repetitions of each of the two patterns each day. Serial-pattern training lasted for 20 days.

Histology. After the completion of serial-pattern learning (approximately 30 days postexposure), the rats were given an overdose of Chloropent; they were then perfused through the heart with saline followed by $10 \%$ neutral buffered Formalin. Frozen sections $(50 \mu \mathrm{m})$ were obtained in the coronal plane, were mounted on slides, and were stained with thionine (Nissl stain).

\section{Results}

TMT-exposed rats showed a slight decline in body weight on Days 5-10 postexposure (rats were not weighed 
on Days 1-4 postexposure). Differences were not found to be significant by an ANOVA ( $p>.05)$. One rat died 3 days after exposure.

Light microscopy revealed thinning of CA1, CA3b, and CA3c pyramidal cell fields of hippocampus following TMT exposure. Pyramidal cell loss was most extensive in the intrahilar CA3c region. The granule cell layer of the dentate gyrus and the pyramidal cell layer of CA2 and CA3a were spared from detectable damage, as were the diffusely scattered intrahilar pyramidal cells of CA4. The photomicrographs of Figure 1 show a coronal section through the brain of a control rat (top panel) and a TMTexposed rat (bottom panel) at approximately the same level of the dorsal hippocampus. These sections are representative of rats from this and the following experiment.

Light-dark discrimination. No significant differences were found between rats' response to BSR just before TMT exposure and Days 5-6 after intubation ( $p>.05)$. Both control and TMT groups leverpressed vigorously for BSR both before and after intubation, and no significant
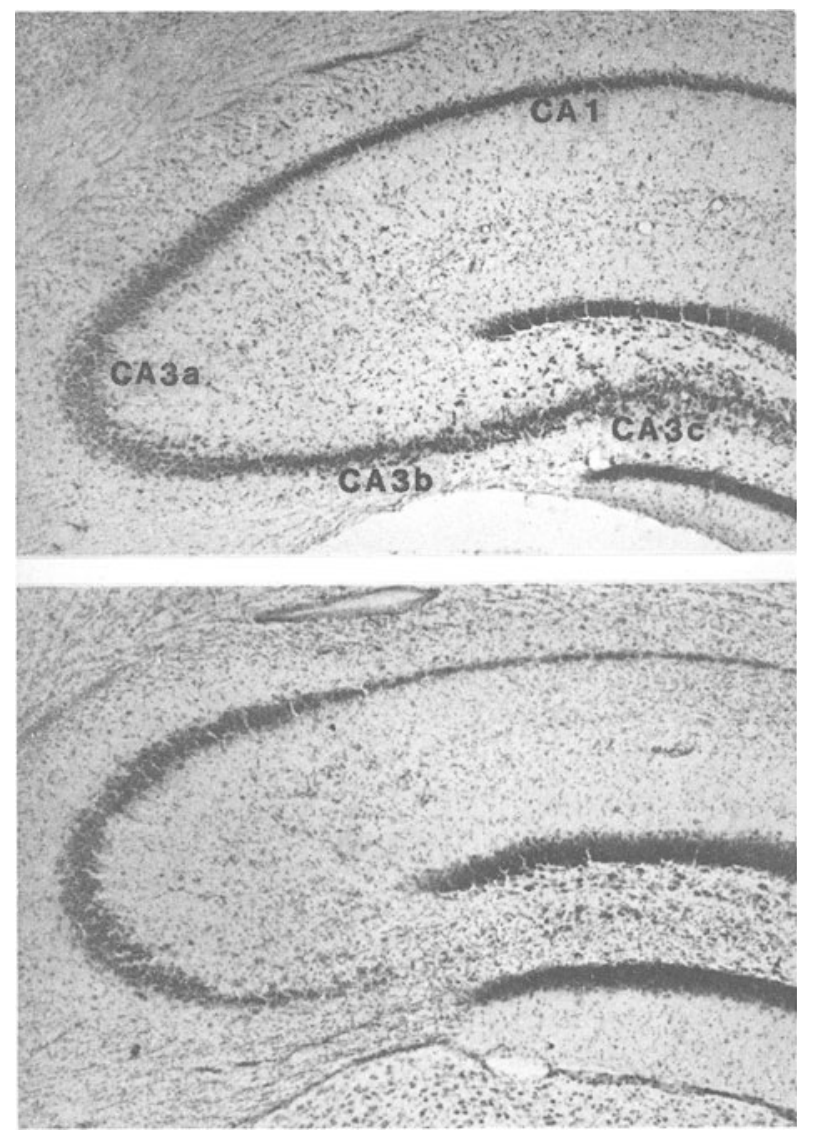

Figure 1. Coronal sections of the brain at the level of the dorsal hippocampus for a representative control rat (top panel) and for a representative rat exposed to $7 \mathrm{mg}$ TMT/kg body weight (bottom panel). For TMT-exposed rats, thinning of hippocampal CA1 and CA3b pyramidal cell fields and virtual destruction of $\mathrm{CA3c}$ in the intrahilar region are evident, but hippocampal CA2, CA3a, and dentate were largely spared. Nissl stain. differences in extinction were found between groups ( $p$ $>.05$ ). That is to say, rats in both groups produced equivalent numbers of reinforced responses during lightson periods and equivalent numbers of nonreinforced responses during lights-out periods before and after intubation. Control rats produced means of 4,826 and 5,319 reinforced responses for the 2 days prior to intubation and for Days 5-6 postexposure, respectively. TMT-exposed rats produced means of 5,027 and 5,443 reinforced responses pre- and postexposure, respectively. An ANOVA conducted on rats' daily total nonreinforced responses during lights-out periods in sessions pre- and postexposure indicated reliable main effects for preversus postexposure $[F(1,6)=26.07, p<.01]$ and days $[F(1,6)=31.09, p<.01]$ and reliable interactions for pre- versus postexposure $\times$ days $[\mathrm{F}(1,6)=21.94, \mathrm{p}<$ $.01]$ and for TMT treatment $\times$ pre- versus postexposure $\times$ days $[F(1,6)=6.50, p<.05]$. Planned comparisons based on the appropriate error term from the ANOVA calculated on these data indicated that both control and TMT-exposed rats produced significantly fewer nonreinforced responses on all subsequent days than on the first training day (ps $<.05$ ), but no significant differences were detected following the first training day $(p>.05)$. An overview of these results shows that control rats produced means of 114 and 52 nonreinforced responses during lights-out intervals pre- and postexposure, respectively; TMT-exposed rats produced means of 98 and 61 nonreinforced responses pre- and postexposure, respectively. For both groups, the greater number of preexposure nonreinforced responses relative to postexposure responses reflects the greater number of nonreinforced responses on the very first day of training. The three-way interaction was the result of significantly more nonreinforced responses produced by controls relative to TMT rats only on the very first day of discrimination training.

Serial-pattern learning. Rats in the control condition rapidly learned to track both their monotonic $20-10-0$ pattern and their nonmonotonic 1-29-0 pattern. That is, they learned to respond fast in anticipation of reinforced trials and slowly, or not at all, in anticipation of nonreinforced 0-pulse trials. TMT-exposed rats also learned their patterns, but they were clearly impaired in learning to track both of their patterns. Although planned comparisons indicated that both groups reliably tracked both patterns, beginning on Day 2 , by responding more slowly on 0 pulse BSR trials than on other trials $(p<.05)$, control rats responded reliably more slowly in anticipation of 0 pulse trials than did TMT-exposed rats on Days 3-14 $(p<.05)$. No significant differences in response latencies were found on Days 15-20 ( $p>$.05). Response latencies for 0 -pulse trials were never significantly different between monotonic and nonmonotonic patterns for either group ( $p>.05$ ), and no significant differences among response latencies for other BSR quantities were found ( $\mathrm{ps}>.05$ ).

Figure 2 shows mean response latency for each BSR quantity that made up both the monotonic (M) 20-10-0 pattern and the nonmonotonic (N) 1-29-0 pattern for con- 


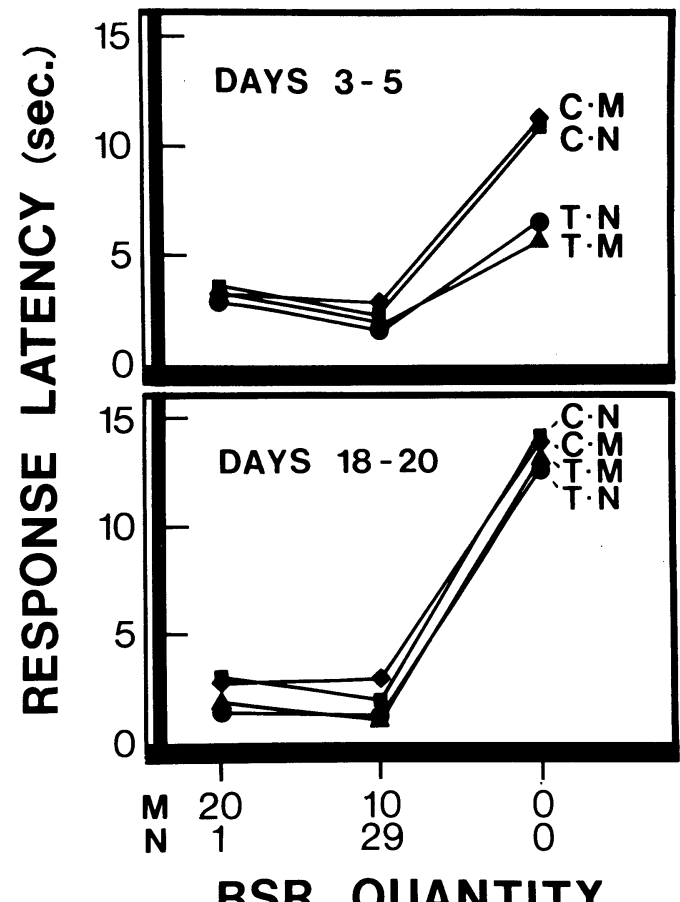

Figure 2. Mean response latency for each quantity of the monotonic (M) 20-10-0 pattern and the nonmonotonic (N) 1-29-0 pattern of Experiment 1 for control (C) and TMT-exposed (T) groups on Days 3-5 (top panel) and Days 18-20 (bottom panel). Each rat received both the monotonic pattern and the nonmonotonic pattern. Control monotonic and nonmonotonic data are represented by filled diamonds and squares, respectively. TMT-exposed monotonic and nonmonotonic data are represented by filled triangles and circles, respectively.

trol (C) and TMT-exposed (T) rats. The top panel of Figure 2 shows results for Days 3-5, the first 3 days in which groups differed in pattern tracking. Control and TMT-exposed groups responded equally fast in anticipation of all reinforced trials and significantly more slowly for 0 -pulse trials $(p<.05)$. In addition, control rats tracked their pattern better than TMT-exposed rats by responding significantly more slowly on 0-pulse trials than did TMT-exposed rats $(p<.05)$. By Days $18-20$, the last 3 days of the experiment, no significant differences were found between the two groups' responses to their patterns $(p>.05)$, as shown in the bottom panel of Figure 2 .

The planned comparisons just reported were based on the appropriate error term from an ANOVA conducted on rats' daily mean response latencies for each quantity of each pattern for the 20 days of the experiment. The ANOVA revealed a significant main effect for serial position $[F(2,12)=103.23, p<.01]$ and significant interactions for days $\times$ serial position $[F(38,228)=20.45$, $\mathrm{p}<.01]$, treatment $\times$ days $\times$ serial position $[\mathrm{F}(38,228)$ $=3.05, \mathrm{p}<.01]$, and days $\times$ patterns $\times$ serial position $[\mathrm{F}(38,228)=1.67, \mathrm{p}<.01]$. Although the days $\times$ patterns $\times$ serial position interaction was significant, planned comparisons failed to detect any significant differences between monotonic and nonmonotonic patterns when daily mean response latencies were pooled across TMT treatment conditions $(p>.05)$. Because the treatment $\times$ days $\times$ patterns $\times$ serial position interaction was not significant, comparisons between response latencies for different BSR quantities between patterns and TMT-treatment conditions were not justified. However, the treatment $X$ days $\times$ serial position interaction was significant. Interpretation of this effect is simplified because the only significant differences in response latencies between treatment conditions were for Trial 3 latencies. Because Trial 3 responses were always followed by the same BSR quantity, namely, the 0-pulse BSR quantity, differences in learning to respond to Trial 3 can be interpreted as differences in learning to anticipate the 0-pulse quantity. In this case, control rats learned to withhold responding on 0-pulse trials better than did TMT-exposed rats from Day 3 until the final days of the experiment.

\section{Discussion}

Rats exposed to TMT 1 week prior to the beginning of serial-pattern learning were clearly impaired relative to controls in their ability to learn to anticipate the 0 -pulse trials that ended both the 20-10-0 pattern and the 1-29-0 pattern. However, although TMT-exposed rats were impaired in their rate of learning relative to controls, control and TMT-exposed rats did not differ in asymptotic performance. Furthermore, rats in both conditions responded with equal vigor for BSR, indicating that the rewarding quality of hypothalamic stimulation was not affected by TMT exposure.

\section{EXPERIMENT 2}

Serial-pattern learning in rats is modulated by pattern length (Fountain, Evensen, \& Hulse, 1983). When patterns are made formally simple, increasing pattern length encourages rats to use a rule-learning strategy. This simplifies pattern learning by reducing the amount of information the rat must encode to learn the pattern. When patterns are made formally complex, increasing pattern length increases the difficulty of the pattern because rule learning cannot be employed as a means of reducing the growing amount of information that must be encoded to learn the pattern (for a summary, see Fountain et al., 1983). Experiment 2 tested the effects of TMT exposure on rats' ability to learn patterns of greater length than three quantities. In Experiment 2, rats were required to learn patterns made up of six quantities arranged in sequential order. The rats received first a formally simple, monotonically decreasing 18-10-6-3-1-0 pattern that alternated throughout testing with a formally complex nonmonotonic 18-1-3-6-10-0 pattern. The former pattern encourages rats to simplify pattern learning by encoding a single rule to learn the pattern, namely, that each successive quantity is "less than" the preceding quantity. No such rule holds for the nonmonotonic pattern, thus rats are forced to learn this pattern by rote. Experiment 2 tested the notion that 
TMT exposure might selectively impair either rule learning or rote learning. In addition, light-dark discrimination sessions were doubled in length, and nonrewarded responses in the intervals between serial patterns were monitored to further substantiate the claim that TMT exposure did not affect performance in the serial-patternlearning task by producing changes in rats' responses to either BSR or nonreward.

\begin{abstract}
Method
Subjects. The subjects were eight naive male hooded rats obtained from Blue Spruce Farms, Altamont, New York. The rats weighed $300-350 \mathrm{~g}$ at the time of surgery. They were approximately 90-110 days of age at the beginning of the experiment. All rats completed the experiment, four rats in each of two conditions. The rats were housed in individual plastic tubs, and were provided free access to food and water in their home cages.

Apparatus. The apparatus was the same as that used in Experiment 1 .

Procedure. The procedures used for implantation of electrodes, intubation, and all leverpress training prior to serial-pattern learning were identical to those used in Experiment 1, with the exception that light-dark discrimination training and testing lasted $1 \mathrm{~h}$ each day rather than $30 \mathrm{~min}$ each day. This procedure constituted a more stringent test of the idea that TMT exposure might affect either rats' responses to BSR or their responses to nonreinforcement. The general procedure for testing rats during the seriallearning phase of Experiment 2 was identical to that used in Experiment 1, with the exception that the two patterns used in Experiment 2 were six BSR quantities long rather than three quantities long. Each day the rats first received an 18-10-6-3-1-0 pattern of BSR quantities follwed by an 18-1-3-6-10-0 pattern. Thereafter, patterns alternated throughout the daily session until the rats received 50 repetitions of each pattern. In addition, nonreinforced leverpresses that occurred during the 1-min lights-out interval between patterns were counted. All other procedures were identical to those used in Experiment 1. Serial-pattern training continued for 14 days. The histological procedures used following serial-pattern training were identical to those used in Experiment 1.
\end{abstract}

\section{Results}

As in Experiment 1, TMT-exposed rats showed a slight decline in body weight. This decline was evident for Days 5-8 postexposure (rats were not weighed on Days 14 postexposure). However, these differences were not found to be significant by an ANOVA ( $p>.05$ ).

Light-dark discrimination. No significant differences were found between rats' response to BSR just before TMT exposure and Days 5-6 after intubation ( $p>.05)$. Both control and TMT groups leverpressed vigorously for BSR both before and after intubation, and no significant differences in extinction were found between groups ( $p$ $>.05$ ). That is to say, rats in both groups produced equivalent numbers of nonreinforced responses during lights-out periods before and after intubation. Control rats produced means of 9,839 and 10,462 reinforced responses for the 2 days prior to intubation and for Days 5-6 postexposure, respectively. TMT-exposed rats produced means of 10,860 and 11,378 reinforced responses pre- and postexposure, respectively. An ANOVA conducted on rats' daily total nonreinforced responses during lights-out periods in sessions pre- and postintubation indicated reliable main effects for pre- versus postexposure $[\mathrm{F}(1,6)=$
$12.65, \mathrm{p}<.05]$ and for days $[\mathrm{F}(1,6)=10.51, \mathrm{p}<.05]$, and a reliable interaction for pre- versus postexposure $X$ days $[F(1,6)=14.84, p<.01]$, but no reliable effects involving TMT treatment ( $\mathrm{ps}>.05$ ). Planned comparisons based on the appropriate error term from the ANOVA calculated on these data indicated that both control and TMT-exposed rats produced significantly fewer nonreinforced responses on all subsequent days than on the first training day (ps $<.05$ ), but no significant differences were detected following the first training day ( $p>$ .05 ). An overview of these results shows that control rats produced means of 131 and 81 nonreinforced responses during lights-out intervals pre- and postexposure, respectively. TMT-exposed rats produced means of 116 and 66 nonreinforced responses pre- and postexposure, respectively. For both groups, the greater number of preexposure nonreinforced responses relative to postexposure responses reflects the greater number of nonreinforced responses on the very first day of training.

Serial-pattern learning. Rats in both the control and TMT-exposed conditions rapidly learned to track their monotonic 18-10-6-3-1-0 pattern, responding fast in anticipation of reinforced trials and slowly in anticipation of nonreinforced trials. Both groups learned to track their nonmonotonic 18-1-3-6-10-0 pattern somewhat more slowly. Planned comparisons showed that control rats tracked their monotonic pattern better than their nonmonotonic pattern on Days 2-3 by responding reliably more slowly in anticipation of the 0-pulse trial of their monotonic pattern than of their nonmonotonic pattern. TMTexposed rats tracked their monotonic pattern better than their nonmonotonic pattern on Days 2-9 (ps < .05). Of greater interest was the finding that TMT-exposed rats tracked their monotonic pattern better than did control rats, but that TMT-exposed rats were impaired in learning their nonmonotonic pattern compared with controls. TMT-exposed and control rats did not differ in learning to anticipate the 0 -pulse quantity of their monotonic pattern. However, TMT-exposed rats responded reliably more slowly in anticipation of the monotonic 1-pulse quantity than of larger monotonic pattern quantities on Days 314 , exclusive of Days 10 and $12(p<.05)$. Control rats never differentiated their response times for the monotonic 1-pulse quantity ( $p>.05$ ). In contrast, TMTexposed rats were impaired in learning their nonmonotonic pattern relative to controls. TMT-exposed rats responded reliably faster on the nonmonotonic 0 -pulse trial than did controls on Days 4-12 ( $p<.05)$.

Figure 3 shows mean response latency for each BSR quantity that made up both the monotonic (M) 18-10-63-1-0 pattern and the nonmonotonic $(\mathrm{N})$ 18-1-3-6-10-0 pattern for control (C) and TMT-exposed (T) rats. The top panel of Figure 3 shows the results for Days 1-3 when differential responding for monotonic and nonmonotonic patterns appeared. Both groups responded reliably more slowly in anticipation of the 0 -pulse quantity of the monotonic pattern than of the nonmonotonic pattern on Days 2 and $3(p<.05)$. Rats in both groups responded reliably 


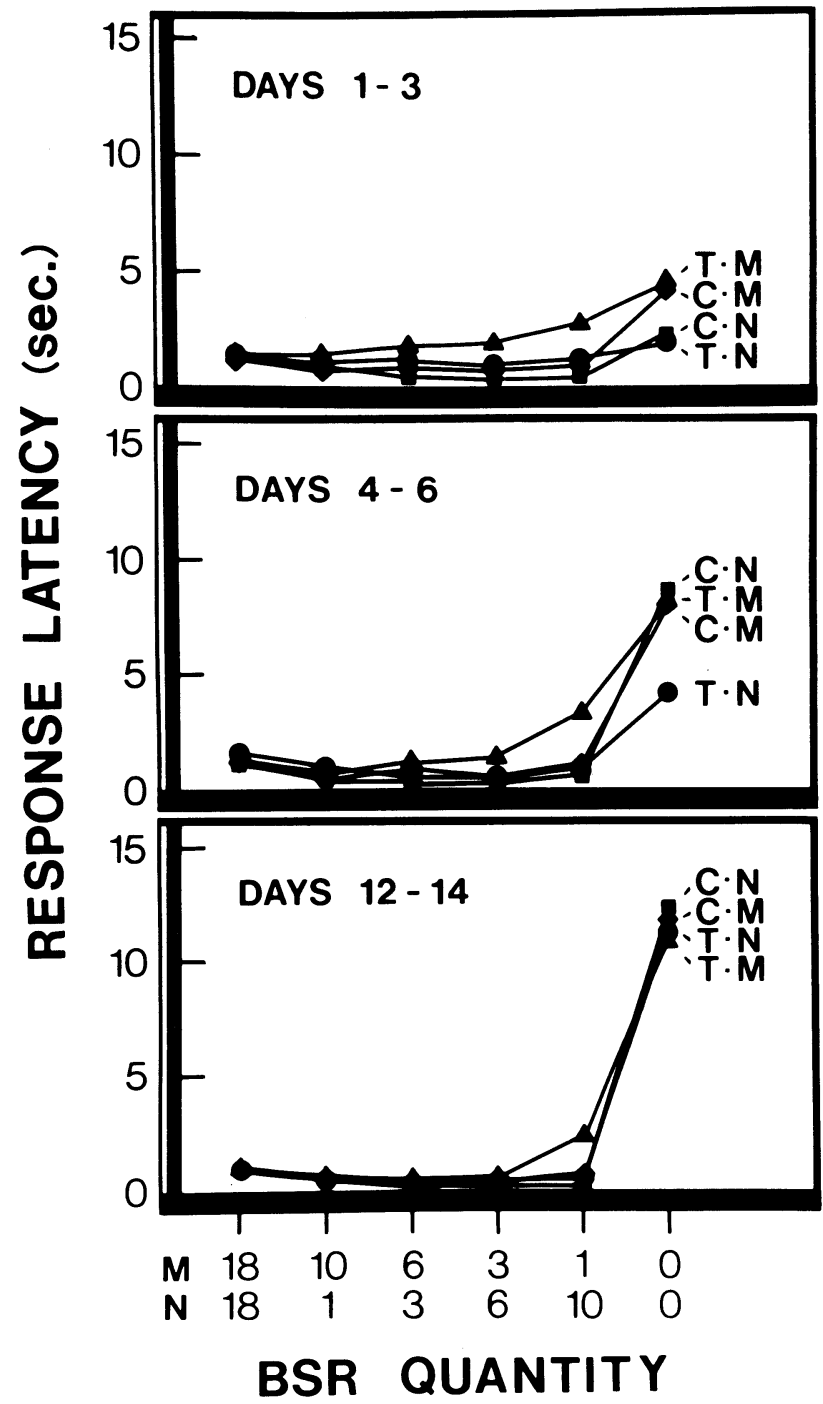

Figure 3. Mean response latency for each quantity of the monotonic (M) 18-10-6-3-1-0 pattern and the nonmonotonic $(N)$ 18-1-36-10-0 pattern of Experiment 2 for control (C) and TMT-exposed (T) groups on Days 1-3 (top panel), Days 4-6 (center panel), and Days 12-14 (bottom panel). Each rat received both the monotonic pattern and the nonmonotonic pattern. Control monotonic and nonmonotonic data are represented by filled diamonds and squares, respectively. TMT-exposed monotonic and nonmonotonic data are represented by filled triangles and circles, respectively.

more slowly in anticipation of the monotonic 0-pulse quantity than of other monotonic quantities beginning on Day 2 $(\mathrm{p}<.05)$. Differential responding for the 0-pulse quantity of the nonmonotonic pattern began for both groups on Day $3(p<.05)$.

The center panel of Figure 3 shows the results for Days 4-6, the first 3 days in which control rats tracked their nonmonotonic pattern reliably better than TMTexposed rats tracked their nonmonotonic pattern. Rats in both groups responded reliably more slowly in anticipation of the 0-pulse quantities of both their monotonic and nonmonotonic patterns relative to larger BSR quantities (ps < .05). However, TMT-exposed rats were impaired in anticipation of their nonmonotonic 0-pulse quantity; they responded faster on the 0-pulse trial of their nonmonotonic pattern than they did on the 0 -pulse trial of their monotonic pattern, and faster than control rats did on the 0-pulse trials of both monotonic and nonmonotonic patterns (ps < .05). Finally, TMT-exposed rats demonstrated better pattern tracking of the monotonic pattern than did controls by responding reliably more slowly in anticipation of the monotonic 1-pulse quantity than did controls $(\mathrm{p}<.05)$. TMT-exposed rats responded reliably more slowly on monotonic 1-pulse trials than either group responded on any other trial of both patterns, exclusive of 0 -pulse trials $(\mathrm{p}<.05)$.

The bottom panel of Figure 3 shows the results of Days 12-14, the last 3 days of the experiment. Rats in both groups responded reliably more slowly in anticipation of 0-pulse quantities of both their patterns than of other quantities $(\mathrm{p}<.05)$, and no significant differences were indicated in comparing response latencies for 0-pulse trials $(\mathrm{p}>$.05). In addition, TMT-exposed rats responded reliably more slowly in anticipation of their monotonic 1-pulse quantity than of other quantities, and more slowly than either group in anticipation of other 1-pulse quantities and larger BSR quantities (ps < .05).

The planned comparisons just reported were based on the appropriate error term from an ANOVA conducted on the rats' daily mean response latencies for each quantity of each pattern for the 14 days of the experiment. The ANOVA revealed significant main effects for days $[\mathrm{F}(13,78)=7.88, \mathrm{p}<.01]$, patterns $[\mathrm{F}(1,6)=12.91$, $\mathrm{p}<.05]$, and serial position $[\mathrm{F}(5,30)=63.63, \mathrm{p}<.01]$. Significant interactions included TMT treatment $\times$ patterns $[F(1,6)=10.84, p<.05]$, days $\times$ patterns $[\mathrm{F}(13,78)=4.64, \mathrm{p}<.01]$, days $\times$ serial position $[\mathrm{F}(65,390)=33.75, \mathrm{p}<.01]$, patterns $\times$ serial position $[\mathrm{F}(5,30)=6.54, \mathrm{p}<.01]$, treatment $\times$ days $\times$ patterns $[\mathrm{F}(13,78)=3.46, \mathrm{p}<.01]$, treatment $\times$ patterns $\times$ serial position $[F(5,30)=5.53, p<.01]$, days $\times$ patterns $\times$ serial position $[\mathrm{F}(65,390)=2.21, \mathrm{p}<.01]$, and treatment $\times$ days $\times$ patterns $\times$ serial position $[\mathrm{F}(65,390)$ $=1.73, \mathrm{p}<.01]$.

Control and TMT-exposed rats produced equivalent numbers of nonreinforced responses in the intervals between patterns for each day of the serial-pattern-learning phase of the experiment. An ANOVA conducted on the rats' daily total nonreinforced responses between patterns for the 14 days of the experiment revealed a reliable main effect for days $[\mathrm{F}(13,78)=3.19, \mathrm{p}<.01]$, but no main effect or interaction involving TMT treatment $(p>.05)$. Rats in both groups showed a sharp decline in nonreinforced responses between patterns over the first 3 days of training (from a mean of 237 responses on Day 1 to a mean of 47 responses on Day 4), and no significant change in performance thereafter.

\section{Discussion}

Rats exposed to TMT 1 week prior to serial-pattern learning were clearly impaired relative to controls in their ability to learn to anticipate the 0-pulse trial that ended their nonmonotonic 18-1-3-6-10-0 pattern. However, 
TMT-exposed rats learned to track their monotonic 1810-6-3-1-0 pattern better than controls as evidenced by comparable anticipation of the 0-pulse BSR quantity and better anticipation of the 1-pulse BSR quantity relative to controls. These results support the idea that TMT exposure impaired the processes involved in rote serial learning, but not the processes mediating rule learning. However, although TMT-exposed rats were impaired in their rate of learning for their nonmonotonic pattern relative to controls, control and TMT-exposed rats did not differ in asymptotic performance for either their monotonic or nonmonotonic pattern. As found in Experiment 1, rats in both conditions responded with equal vigor for BSR. In addition, no differences in extinction were found attributable to TMT exposure at any time during the experiment. These results further support the idea that the rewarding quality of hypothalamic stimulation was not affected by TMT exposure, and that the impairments in performance reflected impairments in learning processes rather than changes in motivational or other systems.

\section{GENERAL DISCUSSION}

Rats exposed to $7.0 \mathrm{mg} / \mathrm{kg}$ TMT showed clear deficits in serial-pattern learning. In Experiment 1, TMT-exposed rats learned to track both a 20-10-0 pattern and a 1-29-0 pattern of BSR quantities more slowly than controls. In Experiment 2, TMT-exposed rats learned a nonmonotonic 18-1-3-6-10-0 pattern of BSR quantities more slowly than controls, but learned their monotonically decreasing 18 10-6-3-1-0 pattern of BSR quantities better than controls. These serial-pattern-learning deficits were independent of other factors that could potentially affect performance. Specifically, in both experiments, rats responded with equal vigor for BSR and showed comparable extinction in response to nonreward outside the context of the serial pattern following TMT exposure. These results thus contradict the view that TMT exposure impairs rats' capacity to inhibit responding (cf. Nation, Bourgeois, Clark, $\&$ Elissalde, 1984). These results also support the contention that the deficits described were, in fact, deficits in the processes involved in serial-pattern learning rather than changes in performance. However, the results showed that asymptotic performance did not differ between treatment groups. One interpretation of the latter finding is that the pattern-learning deficit following TMT exposure was transient. Perhaps TMT-exposed rats' improvement in performance to control levels at asymptote represents some as yet unspecified recovery of function. Alternatively, TMT-exposed rats might be permanently slower learners than controls. A third alternative is that TMT-exposed rats might still be impaired relative to controls even at asymptote, although ceiling effects obscured the deficit in pattern learning. Our experiments did not resolve this issue.

The results of Experiments 1 and 2 are consistent with previous research describing the trimethyltin syndrome in rats. The mortality rate of one rat of the nine exposed in the two experiments is consistent with the findings of previous studies of mortality following an acute dose of $7 \mathrm{mg} / \mathrm{kg}$, especially considering the body weight of the rats involved (Dyer, Walsh, et al., 1982). The failure to find significant decreases in body weight following TMT exposure is also consistent with other data for rats of similar body weight (Dyer, Walsh, et al., 1982). Our dose of $7 \mathrm{mg} / \mathrm{kg}$ TMT was only slightly less than the $\mathrm{LD}_{50}$ of $7.45 \mathrm{mg} / \mathrm{kg}$ established for an acute dose of TMT administered by intubation (Dyer, Walsh, et al., 1982). Although the slope of the lethality $\times$ TMT dosage curve is steep, the putative relationship of initial body weight to lethality allowed us to choose a dose certain to produce neurological alterations (Dyer, Walsh, et al., 1982) with a minimum of decline in body weight (Nation et al., 1984), a minimum of TMT-induced tremor, and a minimum of lethality (Dyer, Walsh, et al., 1982). High, acute doses of TMT ( $7 \mathrm{mg} / \mathrm{kg}$ or greater) administered by intubation reliably produce loss of pyramidal cells within the hippocampal formation, primarily from the CA3b, CA3c, and CA4 pyramidal cell fields (Dyer, Deshields, \& Wonderlin, 1982; Dyer, Walsh, et al., 1982) with occasional thinning of the CA1 pyramidal cell field (Brown et al., 1979; Chang, Wenger, McMillan, \& Dyer, 1983; Swartzwelder et al., 1982). Similarly, using other exposure procedures, such as sequential administration or intraperitoneal injection, doses of $7 \mathrm{mg} / \mathrm{kg}$ TMT produce damage primarily in the hippocampus (Brown et al., 1979; Valdes et al., 1983) with limited damage in other limbic structures and neocortex (Brown et al., 1979). Our histological results paralleled these findings. Thus, we suggest that our serial-pattern-learning deficits are likely the result of limbic system dysfunction. A characterization of the nature of the pattern-learning deficit follows.

\section{The Serial-Pattern-Learning Deficit}

Two current views of rat serial-pattern learning must be considered to understand the nature of the patternlearning deficit produced by TMT exposure. The first view assumes that rats anticipate the reward of a trial by remembering what reward was received on the previous trial as a cue for what will be received on the current trial. This memory-of-prior-reward view (Capaldi \& Molina, 1979) further assumes that the relative discriminability and "generalized reward signal capacity" (Capaldi \& Molina, 1979) of the rewarding events that make up the pattern are the factors most responsible for how difficult to learn a pattern will be. This view predicts that the 1-29-0 pattern of Experiment 1 should be easier to learn than the 20-10-0 pattern because the BSR quantities of the nonmonotonic 1-29-0 pattern are more discriminable than those of the monotonic 20-10-0 pattern. In Experiment 2, the nonmonotonic 18-1-3-6-10-0 pattern should be easier to learn than the monotonic 18-10-6-3-1-0 pattern, following the same logic, because the quantity predicting the 0 -pulse quantity for the nonmonotonic pattern (10 pulses) is a more discriminable cue than the quantity predicting 
0 for the monotonic pattern ( 1 pulse). In addition, because the two largest quantities in the nonmonotonic pattern (18 and 10 pulses) both predict dramatic decreases in reinforcement on the next trial ( 1 and 0 pulses, respectively), inhibitory strength should generalize between the memories of the two predictive quantities, thus producing rapid learning.

The second view of rat serial-pattern learning assumes that rats are sensitive to the structure of patterns and that they search patterns for regularities which they can encode using rules to simplify pattern learning (cf. Hulse, 1978). The implications of this view are seen most clearly by comparing the patterns of Experiment 2 . The monotonic 18-10-6-3-1-0 pattern is formally simple because it can be described by a single "less than" rule. That is to say, each pattern quantity in the sequence is "less than" the immediately preceding quantity. The rat can simplify learning this pattern by encoding some representation of the single "less than" rule rather than memorizing a sequence of associations, as in the memory-of-prior-reward strategy. Here learning a single rule rather than many associations substantially reduces what the rat must remember to respond appropriately to the pattern. This strategy is not as effective, however, for the nonmonotonic pattern of Experiment 2. This pattern is formally complex because no single rule describes all the pairwise relationships of the BSR quantities that make up the 18-1-3-610-0 pattern. Instead, both "less than" and "greater than" rules are required for an adequate description of the pattern. The rule-learning view, then, predicts that learning the monotonic 18-10-6-3-1-0 pattern will be easier than learning the nonmonotonic $18-1-3-6-10-0$ pattern. Note that the rule-learning view predicts just the opposite of what the memory-of-prior-reward view predicts at this point. The rule-learning view is less clear in its predictions for the patterns of Experiment 1. On the one hand, the monotonic $20-10-0$ pattern can be described by a single "less than" rule, whereas the nonmonotonic 1-29-0 pattern cannot be described by a single rule. The rulelearning view should thus predict that the monotonic pattern should be easier to learn than the nonmonotonic pattern. However, as Hulse (1978) pointed out, these patterns are so short that they reduce the advantage of the rule-learning strategy over the memory-of-prior-reward strategy. The rats' choice of strategy in this case may be determined by preference, relative discriminability of pattern elements, or other factors. Previous research using the patterns of Experiments 1 and 2, where the patterns were composed of quantities of food rather than quantities of BSR, showed that the formally simple monotonic 18-10-6-3-1-0 pattern was the easier of the long patterns for rats to learn (Fountain et al., 1983), but the nonmonotonic 1-29-0 pattern was the easier of the short patterns to learn (Capaldi \& Molina, 1979). Thus, the rule-learning view described the results for long patterns, but the memory-of-prior-reward view better described the results for short patterns.
The results from control and TMT-exposed rats support the dichotomy of pattern-learning strategies just outlined. First, control rats in Experiment 1 learned to track their nonmonotonic 1-29-0 pattern somewhat faster than their monotonic 20-10-0 pattern, although this difference was not significant. In contrast, control rats in Experiment 2 learned to track their monotonic 18-10-6-3-1-0 pattern faster than their nonmonotonic 18-1-3-6-10-0 pattern. These results mirror those described above (Capaldi \& Molina, 1979; Fountain et al., 1983) and lend credence to the idea that rats may use different strategies for learning long versus short patterns (cf. Fountain et al., 1983). Second, TMT-exposed rats showed deficits in learning serial patterns in a manner consistent with the idea of a dichotomy of pattern-learning strategies. TMT-exposed rats appeared to be deficient in learning patterns when rule-learning was not the strategy of choice, whereas their rate of pattern learning was comparable to, if not better than, that of controls for the pattern most conducive to a rule-learning strategy. In Experiment 1, TMT-exposed rats learned both of their patterns more slowly than controls. This result is consistent with the idea that rats learned these patterns using the memory-of-prior-reward strategy, or some similar associative strategy, and that it was the processes underlying this strategy that were compromised by TMT exposure. Further evidence for this view is found in Experiment 2, in which TMT-exposed rats learned to track their formally simple monotonic 1810-6-3-1-0 pattern as fast as controls, but were impaired in learning to track their formally complex nonmonotonic 18-1-3-6-10-0 pattern relative to controls. The processes involved in rule learning for the formally simple monotonic pattern were clearly spared. Learning the nonmonotonic pattern, which of necessity was more dependent on impaired associative processes, was disrupted.

Rats' ability to discriminate their patterns also lends credence to the idea that their differential pattern-learning impairment was a function of their adopting different pattern-learning strategies for their two patterns. Experiment 2 clearly showed that TMT-exposed rats readily discriminated pattern elements as belonging to one pattern or the other, yet were impaired in learning only the nonmonotonic $18-1-3-6-10-0$ pattern, not the monotonically decreasing 18-10-6-3-1-0 pattern. Although the 1-pulse BSR quantity preceded, and thus predicted, the 0-pulse quantity in the monotonic 18-10-6-3-1-0 pattern, the 1pulse quantity also preceded the 3-pulse quantity of the nonmonotonic 18-1-3-6-10-0 pattern. Rats showed no detectable confusion between the two patterns; they responded slowly in anticipation of the 0-pulse quantity during monotonic patterns (on the trial following the 1pulse quantity), but responded fast in anticipation of the 3-pulse quantity during nonmonotonic patterns (also on a trial following the 1-pulse quantity). Similarly, the 10pulse quantity preceded, and thus predicted, the 6-pulse quantity of the monotonic 18-10-6-3-1-0 pattern as well as the 0 -pulse quantity of the nonmonotonic $18-1-3-6-10-0$ 
pattern, but rats had no difficulty in distinguishing patterns and organizing their behavior appropriately. Differential impairment, then, was not the result of a breakdown in rats' ability to keep track of which pattern they were receiving. Instead, differential impairment of pattern learning was coincident with pattern organization and reflected differential impairment in processes associated with the rats' acquisition of different representations (Roitblat, 1982) of the formally complex nonmonotonic pattern and the formally simple, rule-based monotonic pattern.

In conclusion, the results of Experiments 1 and 2 are consistent with the idea that, by producing damage in the limbic system, TMT impaired the processes involved in more associative pattern-learning strategies while leaving intact the processes involved in rule learning. Other evidence is also consistent with this general idea. For example, rats with fimbria-fornix lesions are able to remember familiar food quantities received in descending order, but are not able to remember quantities received out of order (Olton, Shapiro, \& Hulse, 1984). This kind of memory impairment and concomitant predisposition to learn and use rules may be related to the distinction made between procedural and declarative knowledge (Cohen \& Squire, 1980; Kesner, 1984; Morris, 1984; Squire \& Cohen, 1984). This distinction is supported by the fact that some human amnesics, including the noted H.M., are able to learn and remember procedural, or rule-based, information about a task while at the same time demonstrating striking deficiencies in the capacity to learn and remember declarative, data-based information (Cohen \& Squire, 1980; Squire \& Cohen, 1984). The results of our experiments fit well with the idea that damage to the limbic system of rats can produce a comparable differentiation of function. Our results are of special significance because they appear to demonstrate a procedural versus declarative dichotomy based on a dichotomy of representation of information presented in the pattern of BSR quantities. In this case, procedural information differs from declarative information in level of abstraction from the stimulus information presented (i.e., BSR quantity); procedural information is some representation of the rulebased organization inherent in the pattern rather than a rote enumeration of the BSR quantities and their order. In addition, our results showed that rats were able to use procedural information that was not restricted to a simple response rule (cf. Morris, 1984). Previous research purporting to demonstrate the declarative versus procedural distinction in animal learning and memory have demonstrated animals' use of data-based information versus response rules (e.g., Gaffan, 1977; Kesner, 1984; Kesner \& Novak, 1982; Morris, 1984). In contrast, our results demonstrate a distinction between rats' use of data-based rote learning processes versus the abstraction and reorganization of information involved when they learn serial patterns by abstracting and encoding a representation of the rule-based structure of their patterns (cf. Hulse, 1978; Hulse \& Dorsky, 1977).

\section{Neurotoxicity and Cognition}

The results of Experiments 1 and 2 support the idea that two pattern-learning processes are differentially affected by limbic system damage resulting from TMT neurotoxicity. However, several questions remain unanswered. For example, our experiments do not address the issue of the permanence or transience of the serial-pattern-learning impairment produced by TMT exposure. Also, one might wonder why TMT-exposed rats in Experiment 1 were not able to use a rule-learning strategy to master their short monotonic pattern, assuming that associative strategies were impaired by TMT exposure. Although these questions remain unanswered, the rule-learning versus associative dichotomy appears to effectively encompass the results requiring explanation.

An interesting implication follows from the result that rule learning, usually considered to be the more complex cognitive function, was not the first system affected by TMT neurotoxicity. One interpretation is that "higher" cognitive functions were spared, whereas more "basic" associative processes were compromised. If so, the idea that complex intellectual functions are more sensitive to toxic insult than other, more "basic" learning and memory functions was not supported. Another idea recently advanced, however, is that the "procedural" memory system is older in evolutionary terms than the "declarative" memory system (Squire \& Cohen, 1984; Thompson et al., 1984). If this is the case, rule-learning processes may represent a more "basic" learning system that can be used independent of associative processes when pattern parameters are optimal (cf. Thompson et al., 1984).

Our results add to the literature demonstrating that toxicity can be expressed in selective learning and memory impairments. Trimethyltin, a relatively selective limbicsystem neurotoxicant, produced a selective serial-patternlearning deficit in rats. The deficit was expressed as an impairment of the rats' capacity to use rote processes to learn serial patterns. In contrast, TMT did not impair rats' ability to encode the rule-based properties of serial patterns. Lesion or stimulation studies, in conjunction with continued investigation of serial learning in rats, should further characterize the nature of the systems underlying the pattern-learning processes dissociated by TMT exposure.

\section{REFERENCES}

Brown, A. W., Aldridge, W. N., Street, B. W., \& Verschoyle, R. D. (1979). The behavioral and neuropathologic sequelae of intoxication by trimethyltin compounds. American Journal of Pathology, 97, 59-82.

CAPAldi, E. J., \& Molina, P. (1979). Element discriminability as a determinant of serial-pattern learning. Animal Learning \& Behavior, 7, 318-322.

Chang, L. W., Wenger, G. R., McMillan, D. E., \& Dyer, R. S. (1983). Species and strain comparison of acute neurotoxic effects of trimethyltin in mice and rats. Neurobehavioral Toxicology \& Teratology, 5, 337-350.

Cohen, N. J., \& SQuire, L. R. (1980). Preserved learning and reten- 
tion of pattern-analyzing skill in amnesia: Dissociation of knowing how and knowing that. Science, 210, 207-210.

Dyer, R. S., Deshields, T. L., \& Wonderlin, W. F. (1982). Trimethyltin-induced changes in gross morphology of the hippocampus. Neurobehavioral Toxicology \& Teratology, 4, 141-147.

Dyer, R. S., Walsh, T. J., Wonderlin, W. F., \& Bercegeay, M. (1982). The trimethyltin syndrome in rats. Neurobehavioral Toxicology \& Teratology, 4, 127-133.

Fountain, S. B., \& ANNAU, Z. (1984). Chunking, sorting, and rulelearning from serial patterns of brain-stimulation reward by rats. Animal Learning \& Behavior, 12, 265-274.

Fountain, S. B., Evensen, J. C., \& Hulse, S. H. (1983). Formal structure and pattern length in serial pattern learning by rats. Animal Learning \& Behavior, 11, 186-192.

Fountain, S. B., Henne, D. R., \& Hulse, S. H. (1984). Phrasing cues and hierarchical organization in serial pattern learning by rats. Journai of Experimental Psychology: Animal Behavior Processes, 10, 30-45.

GAFFAN, D. (1977). Response coding in recall of colors by monkeys. Quarterly Journal of Experimental Psychology, 29, 597-605.

HulSE, S. H. (1978). Cognitive structure and serial pattern learning by animals. In S. H. Hulse, H. Fowler, \& W. K. Honig (Eds.), Cognitive processes in animal behavior (pp. 311-340). Hillsdale, $\mathrm{NJ}$ : Erlbaum.

Hulse, S. H., \& DorsKy, N. P. (1977). Structural complexity as a determinant of serial pattern learning. Learning \& Motivation, 8, 488-506.

KESNER, R. P. (1984). The neurobiology of memory: Implicit and explicit assumptions. In G. Lynch, J. L. McGaugh, \& N. M. Weinberger (Eds.), Neurobiology of learning and memory (pp. 111-118). New York: Guilford.

KeSNER, R. P., \& NovaK, K. J. (1982). Serial position curve in rats: Role of the dorsal hippocampus. Science, 218, 173-174.

MoRrIs, R. G. M. (1984). Is the distinction between procedural and declarative memory useful with respect to animal models of amnesia? In G. Lynch, J. L. McGaugh, \& N. M. Weinberger (Eds.), Neurobiology of learning and memory (pp. 119-124). New York: Guilford.

Nation, J. R., Bourgeois, A. E., Clark, D. E., \& Elissalde, M. (1984). Effects of acute trimethyltin exposure on appetitive acquisi- tion and extinction performance in the adult rat. Behavioral Neuroscience, 98, 919-924.

Olton, D. S., Shapiro, M. L., \& Hulse, S. H. (1984). Working memory and serial patterns. In H. L. Roitblat, T. G. Bever, \& H. S. Terrace (Eds.), Animal cognition (pp. 171-182). Hillsdale, NJ: Erlbaum.

Roitblat, H. L. (1982). The meaning of representation in animal memory. Behavioral \& Brain Sciences, 5, 353-371.

RupPert, P. W., Walsh, T. J., ReIter, L. W., \& Dyer, R. S. (1982). Trimethyltin-induced hyperactivity: Time course and pattern. Neurobehavioral Toxicology \& Teratology, 4, 135-139.

SQUiRE, L. R., \& COHEN, N. J. (1984). Human memory and amnesia. In G. Lynch, J. L. McGaugh, \& N. M. Weinberger (Eds.), Neurobiology of learning and memory (pp. 3-64). New York: Guilford.

Swartzwelder, H. S., Hepler, J., Holahan, W., King, S. E., Levereuz, H. A., Miller, P. A., \& MYers, R. D. (1982). Impaired maze performance in the rat caused by trimethyltin treatment: Problemsolving deficits and perseveration. Neurobehavioral Toxicology \& Teratology, 4, 169-176.

Thompson, R. F., Clark, G. A., Donegan, N. H., Lavond, D. G., Lincoln, J. S., Madden, J., IV, Mamounas, L. A., Mauk, M. D., McCormicK, D. A., \& ThOMPSON, J. K. (1984). Neuronal substrates of learning and memory: A "multiple-trace" view. In G. Lynch, J. L. McGaugh, \& N. M. Weinberger (Eds.), Neurobiology of learning and memory (pp. 137-164). New York: Guilford.

Valdes, J. J., Mactutus, C. F., Santos-Anderson, R. M., Dawson, R., \& ANNAU, Z. (1983). Selective neurochemical and histological lesions in rat hippocampus following chronic trimethyltin exposure. Neurobehavioral Toxicology \& Teratology, 5, 357-361.

Walsh, T. J., Gallagher, M., Bostock, E., \& Dyer, R. S. (1982). Trimethyltin impairs retention of a passive avoidance task. Neurobehavioral Toxicology \& Teratology, 4, 163-167.

Walsh, T. J., Miller, D. B., \& DYeR, R. S. (1982). Trimethyltin, a selective limbic system neurotoxicant, impairs radial-arm maze performance. Neurobehavioral Toxicology \& Teratology, 4, 177-183.

(Manuscript received April 15, 1985; revision accepted for publication August 28, 1985.) 